\title{
Lista de Pareceristas
}

Angélica Roxinsky de Carvalho

Paulo Mengalço Silva Junior

Natália Tavares de Azevedo

Felipe Bueno Amaral

Fernando Mendes Coelho

Raíssa Regina Brugiato Rodrigues

Melissa Gabriela Lopes Barcellos

Coimbra
Victor Miguel Castillo de Macedo Marcelo Tadvald Batista

Marcos Vinicius de Freitas Reis

Marcelo Bordin

Renan Theodoro de Oliveira

Benito Eduardo Maeso

Eloyluz de Sousa Moreira 